\title{
THE EXTENSIVE AIR SHOWER EXPERIMENT KASCADE-GRANDE
}

DONGHWA KANG ${ }^{a *}$, W. D. APEL ${ }^{b}$, J. C. ARTEAGA ${ }^{a}$, F. BADEA $^{b}, \mathrm{~K}$ BEKK $^{b}$, M. BERTAINA ${ }^{c}$, J. BLÜMER ${ }^{a, b}$, H. BOZDOG ${ }^{b}$, I. M. BRANCUS ${ }^{d}$, M. BRÜGGEMANN ${ }^{e}$, P. BUCHHOLZ ${ }^{e}$, E. CANTONI ${ }^{c}$, A. CHIAVASSA ${ }^{c}$, F. COSSAVELLA $^{a}$, K. DAUMILLER $^{b}$,

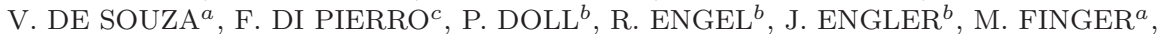
D. FUHRMANN ${ }^{f}$, P. L. GHIA ${ }^{g}$, H. J. GILS ${ }^{b}$, R. GLASSTETTER ${ }^{f}$, C. GRUPEN ${ }^{e}$,

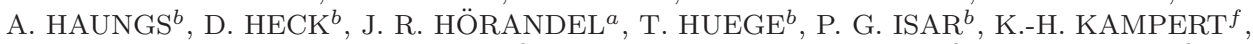
D. KICKELBICK ${ }^{e}$, H. O. KLAGES ${ }^{b}$, Y. KOLOTAEV ${ }^{e},{\text { P. } \text { ŁUCZAK }^{h}, \text { H. J. MATHES }}^{b}$, H. J. MAYER ${ }^{b}$, J. MILKE ${ }^{b}$, B. MITRICA ${ }^{d}$, C. MORELLO ${ }^{g}$, G. NAVARRA $^{c}$, S. NEHLS $^{b}$,

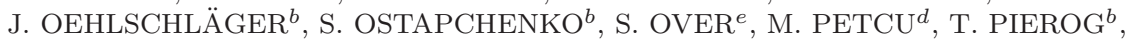
H. REBEL ${ }^{b}$, M. ROTH ${ }^{b}$, G. SCHATZ ${ }^{b}$, H. SCHIELER ${ }^{b}$, F. SCHRÖDER ${ }^{b}$, O. SIMA $^{i}$, M. STÜMPERT ${ }^{a}$, G. TOMA ${ }^{d}$, G. C. TRINCHERO ${ }^{g}$, H. ULRICH ${ }^{b}$, J. VAN BUREN ${ }^{b}$, W. WALKOWIAK ${ }^{e}$, A. WEINDL ${ }^{b}$, J. WOCHELE ${ }^{b}$, M. WOMMER $^{b}$, J. ZABIEROWSKI $^{h}$

${ }^{a}$ Institut für Experimentelle Kernphysik, Universität Karlsruhe, 76021 Karlsruhe, Germany

${ }^{b}$ Institut für Kernphysik, Forschungszentrum Karlsruhe, 76021 Karlsruhe, Germany ${ }^{c}$ Diparimento di Fisica Generale dell'Università, 10125 Torino, Italy

${ }^{d}$ National Institute of Physics and Nuclear Engineering, 7690 Bucharest, Romania ${ }^{e}$ Fachbereich Physik, Universität Siegen, 57068 Siegen, Germany ${ }^{f}$ Fachbereich Physik, Universität Wuppertal, 42097 Wuppertal, Germany ${ }^{g}$ Istituto di Fisica dello Spazio Interplanetario, INAF, 10133 Torino, Italy ${ }^{h}$ Soltan Institute for Nuclear Studies, 90950 Lodz, Poland

${ }^{i}$ Department of Physics, University Bucharest, 76900 Bucharest, Romania *e-mail: donghwa.kang@ik.fzk.de

\begin{abstract}
The extensive air shower experiment KASCADE-Grande (KArlsruhe Shower Core and Array DEtector and Grande array) is located on site of the Forschungszentrum Karlsruhe in Germany. The original KASCADE experiment consisted of a densely packed scintillator array with unshielded and shielded detectors for the measurement of the electromagnetic and muonic shower component independently, as well as muon tracking devices and a hadron calorimeter. The Grande array as an extension of KASCADE consists of 37 scintillation detector stations covering an area of $700 \times 700 \mathrm{~m}^{2}$. The main goal for the combined measurements of KASCADE and Grande is the investigation of the energy spectrum and composition of primary cosmic rays in the energy range of $10^{16}$ to $10^{18} \mathrm{eV}$. In this paper an overview of the KASCADE-Grande experiment and recent results will be presented.
\end{abstract}

Keywords: Cosmic rays; KASCADE-Grande; constant intensity cut method.

PACS Nos.: 96.50.S-, 96.50.sd, 96.50.sb

\section{Introduction}

Since the discovery of cosmic rays investigations of ultra-high energy cosmic rays in many aspects have been done by various experiments. Nevertheless, their sources 


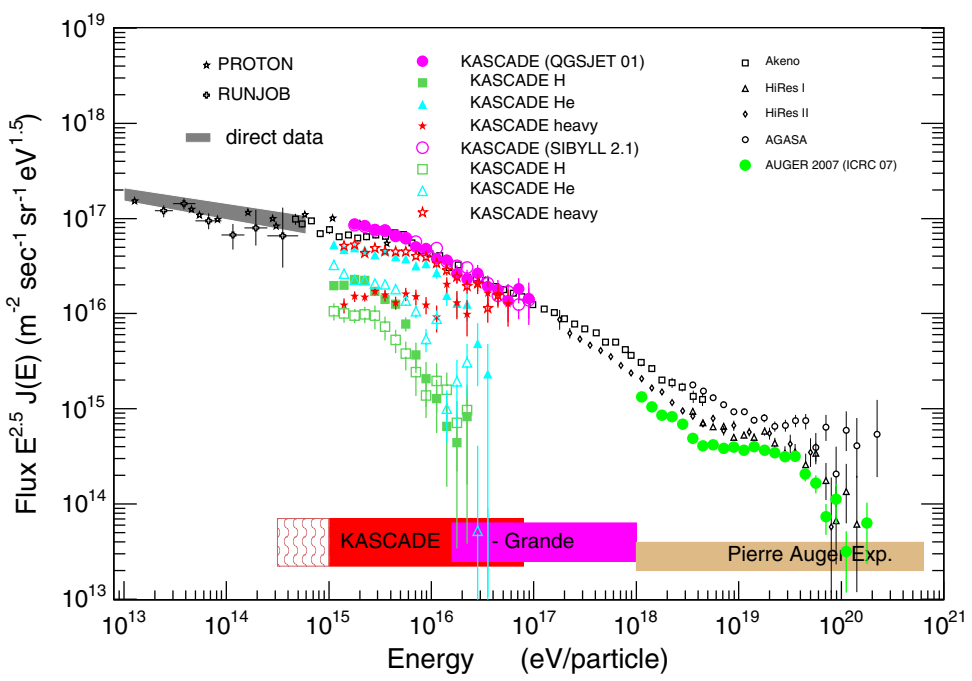

Fig. 1. (Color online) The primary cosmic ray energy spectrum measured by several experiments.

and acceleration mechanisms are still not well known. The all-particle energy spectrum of cosmic rays measured by different experiments is shown in Fig. 1, where the flux is multiplied by a factor of $E^{2.5}$ in order to see structures of the spectrum in more detail. Generally, the energy spectrum follows a power law $d N / d E \propto E^{\gamma}$ with a spectral index of $\gamma$ around -3 .

Particles up to the energy of about $10^{14} \mathrm{eV}$ can be measured directly by balloon or satellite experiments, whereas for higher energies direct measurements cannot provide data with sufficient statistics due to their small sensitive detection area and exposure time. Experiments thus have to observe the cosmic rays indirectly by measuring extensive air showers.

The prominent feature in the energy spectrum is the so-called knee at around $(3-5) \times 10^{15} \mathrm{eV}$ where the spectral index changes from about -2.7 to -3.1 . The origin of the knee is still an unsolved problem, but the general explanation is that the steepening of the spectrum is due to the breakdown of galactic acceleration mechanisms of the cosmic rays ${ }^{1}$ or due to propagation effects. Generally, supernova remnants are believed to be the sources for cosmic rays from about $10 \mathrm{TeV}$ up to the knee with acceleration of the particle by the first order Fermi mechanism: ${ }^{2}$ The cosmic ray particles gain their final energy by many interactions each with a small increase of the energy emitted by supernova explosions. The maximum attainable energy of charged particles is given by $E_{\max } \propto Z \cdot B \cdot R$, where $Z$ is the cosmic ray particle charge, $B$ and $R$ are the magnetic field strength and the size of the acceleration region, respectively. Hence, the energy positions of the knees for different cosmic ray primaries would be expected to depend on their atomic number $Z$. On the contrary, particle physics models generally predict that the knee position 
with respect to the type of the primary particles depends on the atomic mass $A$ rather than the atomic number $Z$. The reason is that the reaction mechanism is followed by the energy per nucleon rather than by the total particle energy of cosmic rays. However, in any of these models, light particles drop out of the spectrum first, so that the energy position of the knee is expected to vary from light to heavy elements, i.e. protons would steepen first, then helium, then CNO. Following that, the heaviest element to be steepened would be the iron. ${ }^{3,4,5}$

Therefore, the main goal of KASCADE-Grande is the observation of the so-called iron-knee in the cosmic ray energy spectrum. It is expected at around $10^{17} \mathrm{eV}$ following previous KASCADE observations ${ }^{6}$ (Fig. 1). In addition, KASCADE-Grande will allow investigations in detail the elemental composition giving the possibility to distinguish between astrophysical models for the transition region from galactic to extragalactic origin of cosmic rays.

\section{The KASCADE-Grande Experiment}

The KASCADE-Grande is an extensive air shower experiment located at Forschungszentrum Karlsruhe (49.1 ${ }^{\circ}$ north, $8.4^{\circ}$ east, $110 \mathrm{~m}$ above sea level). The KASCADE-Grande experiment has been designed to measure primary cosmic rays in the energy range of $10^{16}$ to $10^{18} \mathrm{eV}$. The main detector components of KASCADE-Grande are the KASCADE array, the Grande array, and the muon detection devices. ${ }^{7}$

The KASCADE array consists of 252 scintillator detector stations with unshielded and shielded detectors located on a grid of $200 \times 200 \mathrm{~m}^{2}$ for the measurement of the electromagnetic and muonic shower $\left(E_{\mu}>230 \mathrm{MeV}\right)$ components independently. ${ }^{8}$ The excellent time resolution of these detectors allows reconstructions of the arrival directions of the showers for searching of large scale anisotropies as well as of cosmic ray point sources. ${ }^{9,10}$ The KASCADE array is optimized to measure extensive air showers in the energy range of $10^{14}$ to $8 \times 10^{16} \mathrm{eV}$. The muon tracking detector covering an area of $128 \mathrm{~m}^{2}$ measures the incident angles of muons $\left(E_{\mu}>800 \mathrm{MeV}\right)$ with an excellent angular resolution. These measurements provide a sensitivity to the longitudinal development of the showers. In addition, muon detector of approximately $120 \mathrm{~m}^{2}$ sensitive area provides information on muons with energies above $2.4 \mathrm{GeV} .^{11}$

The Grande array covering an area of $700 \times 700 \mathrm{~m}^{2}$ extends cosmic ray measurements up to primary energies of 1 EeV. ${ }^{12,13,14}$ It comprises 37 scintillation detector stations located on a hexagonal grid with an average spacing of $137 \mathrm{~m}$ for the measurements of the charged shower component. Each of the detector stations is equipped with plastic scintillator sheets covering a total area of $10 \mathrm{~m}^{2}$. The stations contain 16 photomultipliers each providing a dynamic range up to 30000 charged particles per station for the reconstruction of particle densities and timing measurements. The timing accuracy of Grande stations allows also for the Grande 

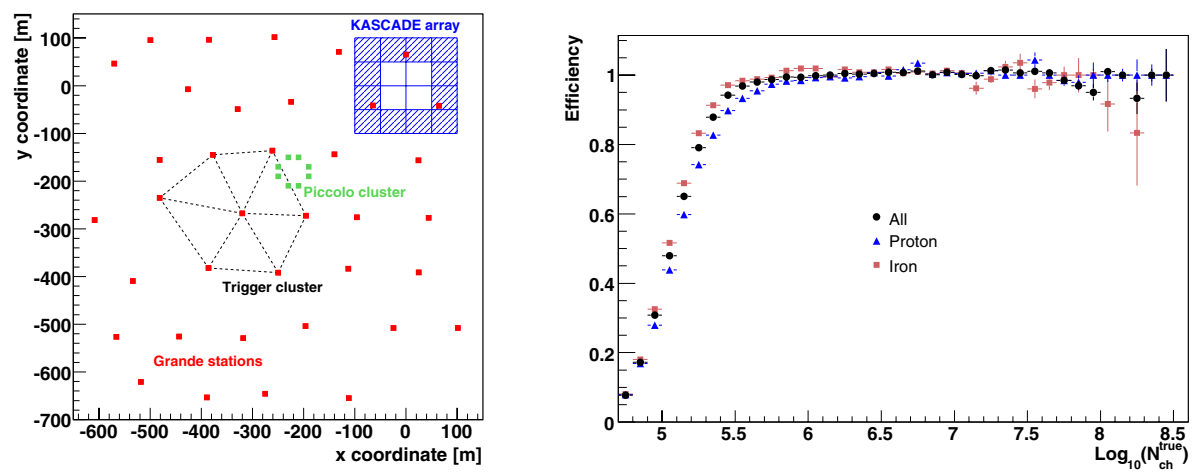

Fig. 2. (Color online) Schematic view of the KASCADE-Grande experiment with 37 Grande, 8 Piccolo stations (left). An example of the 18 hexagonal trigger cluster is shown as well. Trigger and reconstruction efficiency as a function of the number of charged particles for different primary particles (right).

array an excellent angular resolution. The signals are amplified inside the Grande stations, and digitized by peak sensitive ADCs after transmission to a central data acquisition station. Grande is electronically subdivided in 18 hexagonal trigger clusters formed by six external and one central stations. A trigger signal is build when all stations in a hexagon are fired, and its total rate is about $0.5 \mathrm{~Hz}$. An additional cluster, named Piccolo, close to the center of KASCADE-Grande has been built to provide a fast trigger to the muon devices at KASCADE to ensure fully efficient measurements for showers with cores located far from the KASCADE array. Piccolo consists of 8 stations equipped with $10 \mathrm{~m}^{2}$ of plastic scintillator each, and trigger rate of Piccolo is about $0.3 \mathrm{~Hz}$.

Fig. 2 represents a schematic view of KASCADE-Grande with all components. The trigger and reconstruction efficiency of KASCADE-Grande as a function of the shower size, i.e. the number of charged particles, is demonstrated in Fig. 2 (right) as well. Full efficiency is reached at the number of charged particles of around $10^{6}$, which approximately corresponds to a primary energy of $10^{16} \mathrm{eV}$, so that a large overlap for cross-checks with KASCADE is attained. The limit at high energies is due to the restricted area of the Grande array.

\section{Reconstruction Accuracies}

The primary energy of cosmic rays are reconstructed by the observed electron and muon numbers at ground. While the Grande detectors are sensitive to all charged particles, the KASCADE detectors measure separately the electromagnetic and muonic components due to the shielding above the muon counters. Therefore, the shower core position, the arrival direction, and the total number of charged particles in the shower are reconstructed from Grande array data, whereas the total number of muons is extracted from the data of the KASCADE muon detectors. The lateral 

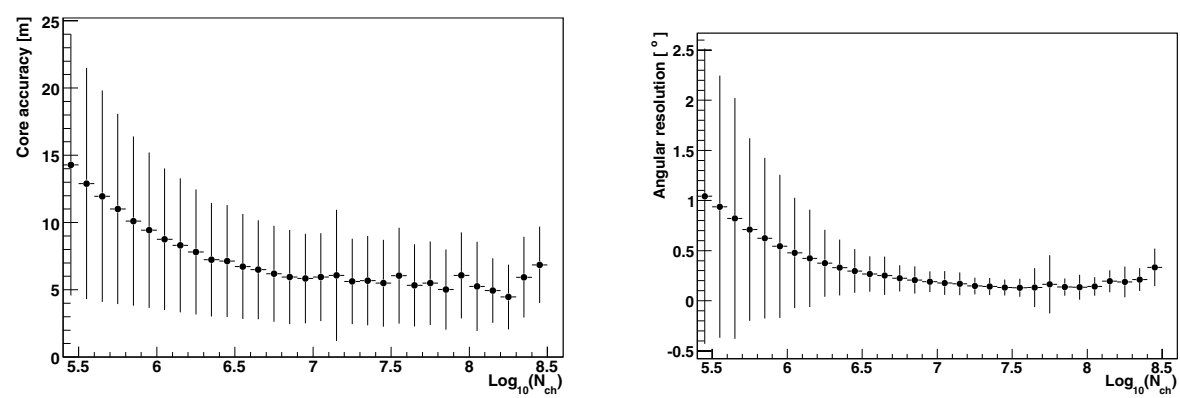

Fig. 3. Reconstruction accuracies of the core position (left) and the arrival direction (right) as a function of the true number of charged particles. The error bars represent the spread of the distribution, i.e. statistical errors, for both cases.

density distribution of the charged particles is described by the modified NKG function, named after J. Nishimura, K. Kamata, and K. Greisen: ${ }^{15}$

$$
\rho_{c h}=N_{c h} \cdot C(s) \cdot\left(\frac{r}{r_{0}}\right)^{s-\alpha}\left(1+\frac{r}{r_{0}}\right)^{s-\beta},
$$

with the normalization factor $C(s)$, the shower size $N_{c h}$, and the shower age $s$. Performing CORSIKA air shower simulations ${ }^{16}$ including the detector response of the Grande array, the parameters $\alpha=1.6, \beta=3.4$, and $r_{0}=30 \mathrm{~m}$ were optimized for the lateral density distribution of KASCADE-Grande. ${ }^{17}$ Fig. 3 shows reconstruction accuracies of shower core position and arrival direction. In order to avoid misreconstruction effects of shower core positions on the border of the Grande array, a fiducial area of about $0.2 \mathrm{~km}^{2}$ centered in the middle of the Grande array is chosen. Above a threshold of $10^{6}$ charged particles, the reconstruction accuracies of the core position and the arrival direction are better than $8 \mathrm{~m}$ and $0.5^{\circ}$, respectively, for zenith angles below $40^{\circ}$. The statistical uncertainty of the shower size is of the order of $20 \%$ for the total number of charged particles (Fig. 4 left panel). All important parameters are well reconstructed with sufficient accuracies for the further relevant physics analysis.

\section{Data Analysis}

KASCADE-Grande has started combined data acquisition with all detector components since the end of 2003. The data presented here were taken from December 2003 to June 2008. It corresponds to the effective measuring time of 803 days, where all components of KASCADE and KASCADE-Grande were operating without failures in data acquisition. In the following preliminary analysis, all showers with zenith angles smaller than $40^{\circ}$ have been analyzed. After some quality cuts approximately 10 million events are available for the physics analysis. 

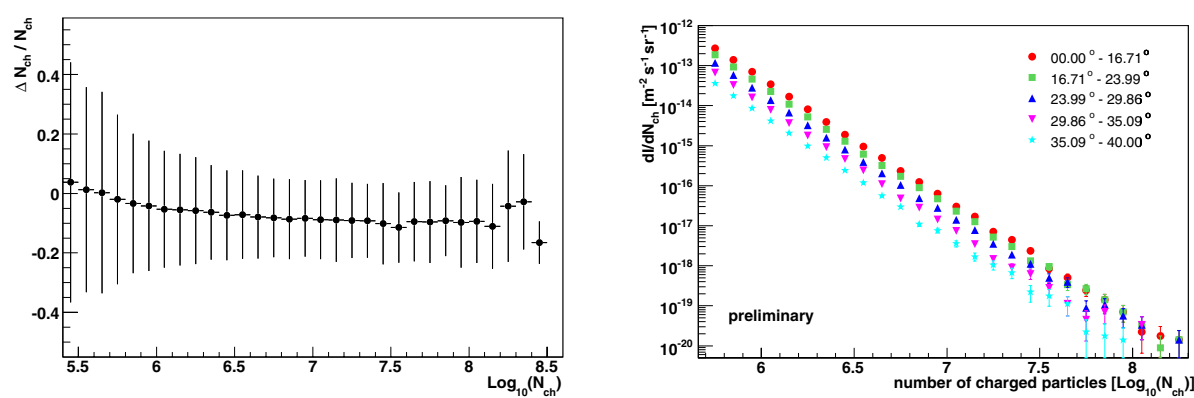

Fig. 4. Reconstruction accuracy of the reconstructed number of charged particles as a function of the true number of charged particles (left). Differential shower size spectra of the number of charged particles for different zenith angle ranges (right).

In order to determine the all-particle energy spectrum, the so-called constant intensity cut method was introduced. This method can be applied with two hypotheses, where the cosmic rays arrive uniformly from all directions and the flux of cosmic rays is distributed isotropically. These assumptions mean that the primary energy of a cosmic ray particle corresponds to a certain intensity regardless of its arrival direction. In KASCADE-Grande, an isotropic distribution is assumed in the considered energy range up to $10^{18} \mathrm{eV}$, so that it allows us to apply the constant intensity method to the KASCADE-Grande data. The first step is to generate shower size spectra, which means the intensity as a function of the number of charged particles $N_{c h}$ for different zenith angular bins. Fig. 4 demonstrates the differential flux normalized by the effective area, the effective measuring time, and the solid angle. Integrating this differential flux above a certain shower size, the intensity should be constant due to the assumption of the uniform intensity distribution when binned in $\cos ^{2} \theta$. The next step is to apply the constant intensity cuts on the integral spectrum. For a given intensity, the number of charged particles is calculated for each zenith angle range. The intensity cut is mostly located in between two neighboring points of the distributions, and thus the values of the corresponding shower size are estimated by interpolation between these two points. From the values of $N_{c h}$ we obtain the so-called attenuation curves (Fig. 5 left panel). It represents how the number of charged particles for a given intensity attenuates through the atmosphere with increasing zenith angle, i.e. with increasing atmospheric depth. Each curve is individually fitted by a second-degree polynomial function, where the reference angle $20^{\circ}$ is chosen by the mean value from a Gaussian fit to the zenith angle distribution. Using one single curve located in the region of full efficiency with the low $\chi^{2}$ value from the fit, the number of charged particles is corrected for the reference zenith angle event by event. The zenith angle dependence of the number of charged particles was therefore eliminated by applying constant intensity cuts. In order to determine the energy conversion relation between the number of charged particles 

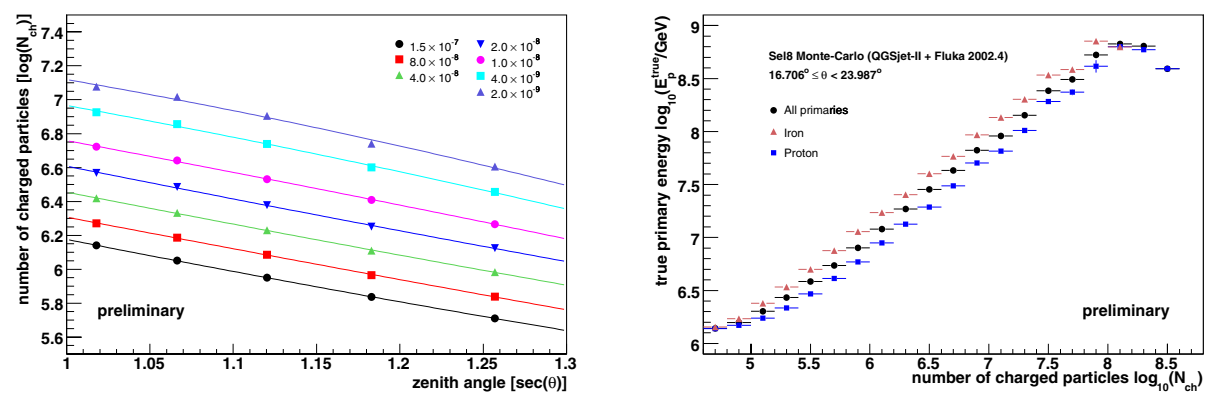

Fig. 5. (Color online) The number of charged particles resulting from the constant intensity cuts as a function of the zenith angle (left). Profile histograms of the true primary energy as a function of the number of charged particles for mixed, proton and iron components (right).

$N_{c h}$ and the primary energy $E_{p}$, the Monte-Carlo simulations were used. Extensive air showers were simulated using the program CORSIKA with QGSjetII ${ }^{18}$ and FLUKA $^{19}$ as hadronic interaction models, including full simulations of the detector response based on GEANT3. The simulated data sets contain air shower events for five different primary mass groups: proton, helium, carbon, silicon, and iron. An equal fraction of $20 \%$ for each group is assumed for a mixed composition. For the simulation, events around the reference zenith angle ranges are selected to reduce systematic effects. The profile histogram of the primary energy as a function of the number of charged particles for a mixed composition is shown in Fig. 5. Assuming a linear dependence, the correlation between the primary energy and the number of charged particles is obtained, where the fit is applied in the range of full trigger and reconstruction efficiencies. The same procedure are performed for pure proton and iron showers. Using these correlations the energy spectra for all primary particles will be obtained. Statistical uncertainties of the reconstructed primary energy spectrum are rather small. Systematic uncertainties of this reconstruction method are presently under investigations. In general, the systematic uncertainties are estimated to be in the order of about 20-30\% in the full energy range under study.

\section{Conclusions and Outlook}

The air shower experiment KASCADE-Grande measures cosmic rays in the energy range of $10^{16}-10^{18} \mathrm{eV}$. The multi detector setup of KASCADE-Grande allows to reconstruct charged particles, electron and muon numbers of the showers separately and with high accuracy. In the present contribution a method was presented to reconstruct the all-particle energy spectrum by means of the charged particle component of the EAS measured by Grande. It could be shown that this method allows to reconstruct the energy spectrum with an accuracy of about 20-30\%. Further checks are currently being performed to reduce the systematic uncertainties 
on the energy estimation, e.g. among others the improvement of the accuracies of the number of charged particles at high energies by optimizing the reconstruction procedures. The measurements of KASCADE-Grande will contribute to solve the astrophysical questions on the existence of the expected iron-knee.

\section{Acknowledgments}

The author would like to thank CosPA2008 organizing committee for their warm hospitality. The KASCADE-Grande experiment is supported by the German Federal Ministry of Education and Research, the MIUR and INAF of Italy, the Polish Ministry of Science and Higher Education and the Romanian Ministry of Education and Research.

\section{References}

1. A. M. Hillas, J. Phys. 47, 168 (2006).

2. Enrico Fermi, Phys. Rev. 75, 1169 (1949).

3. A. Haungs, H. Rebel and M. Roth, Rep. Prog. Phys. 66, 1145 (2003).

4. V. Berezinsky et al., Phys. Rev. D 74, 043005 (2006).

5. R. Aloisio et al., Astrop. Phys. 27, 76 (2007).

6. T. Antoni et al., KASCADE Collaboration, Astrop. Phys. 24, 1 (2005).

7. G. Navarra et al., KASCADE-Grande Collaboration, Nucl. Instr. Meth. A 518, 207 (2004).

8. T. Antoni et al., KASCADE Collaboration, Nucl. Instr. Meth. A 513, 490 (2003).

9. T. Antoni et al., KASCADE Collaboration, Astrophys. J. 608, 865 (2004).

10. T. Antoni et al., KASCADE Collaboration, Astrophys. J. 604, 687 (2004).

11. P. Doll et al., Nucl. Instr. Meth. A 488, 517 (2002).

12. A. Haungs et al., KASCADE-Grande Collaboration, Proc. of 30th ICRC, Vol. 4, pp. 219 (2007).

13. K.-H. Kampert et al., KASCADE-Grande Collaboration, Nucl. Phys. B (Proc. Suppl.) 122, 422 (2003).

14. M. Bertaina et al., KASCADE-Grande Collaboration, Nucl. Instr. Meth. A 588, 162 (2008).

15. K. Greisen, Porg. Cosmic Ray Phys. III 1-141 (1956).

16. D. Heck et al., Rep. FZKA 6019, Forschungszentrum Karlsruhe (1998).

17. F. Di Pierro et al., KASCADE-Grande Collaboration, Proc. of 30th ICRC, Vol. 4, pp. 195 (2007).

18. S.S. Ostapchenko, Phys. Rev. D 74, 014026 (2006).

19. A. Fassò et al., FLUKA: a multi-particle transport code, Report CERN-2005-10 (2005), INFN/TC_05/11, SLAC-R-773. 